\title{
Die Sporenbildung des Milzbrandes bei Anaërobiose.
}

\author{
Erwiderang.
}

Von

\author{
Apotheker Dr. Richard Weil, \\ Assiatenten am stastl. hygien. Institut Hamburg.
}

In Bd. XXXV dieser Zeitschrift èrschien aus dem hygienischen Laboratorium des königlich württembergischen Medicinalcollegiums eine Arbeit von Hrn. Oberarzt Dr. Klett, die, falls sie einer sachlichen Kritik stand zu halten vermag, eine völlige Reform unserer Anschauungen über Anaërobiose herbeiführen müsste. Auffallend ist allerdings die Thatsache, dass der eigene Text mit manchen in den Tabellen niedergelegten Daten im directen Widerspruch steht.

Es sei meine Aufgabe, zuerst den Text für sich, alsdann den Text in Beziehung $z \mathfrak{u}$ den Tabellen eingehend zu untersuchen.

In einer Zusammenstellung der neueren Arbeiten bespricht Klett auch die meinige und beginnt: „Im letzten Jahre veröffentlichte Weil eine Arbeit über die Biologie des Milzbrandes und wies darin nach, dass bei der Sporenbildung jedenfalls nicht die Erschöpfung des Nährbodens von ausschlaggebendem Einfluss sein könne, da dieselbe Bouillon, die nach reichlicher Bildung von Milzbrandsporen mittels Filtration durch ein Chamberlandfilter keimfrei gemacht war, eine reichliche Entwickelung zeigte, wenn sie mit denselben Sporen von neuem infieirt und bei geeigneter Temperatur im Brutschrank gehalten wurde."

Ich ${ }^{1}$ wies also nach, dass Sporen in demselben Nährmaterial auszukeimen vermögen, in dem sie vorher gebildet wurden.

Was hat denn die Auskeimung mit der Sporenbildung zu thun? Wie kommt Klett zu der unrichtigen Auffassung, ich hätte nachgewiesen, dass die Erschöpfung des Nährbodens bei dër Sporenbildung jedenfalls nicht von aussehlaggebendem Einfluss sein könne?

Einer der Schlusssätze meiner Arbeit lautet: Der atmosphärische Sauerstoff übt keinen specifischen Einfluss anf das Zustandekommen der Dauerformen aus. Die Milzbrandbacillen bilden in geeigneten Nährmedien

1 Weil, Zur Biologie des Milzbrandes. Archiv für Hygiene. Bd. XXXV. 
auch unter a naërobiontischen Bedingungen Sporen von beinahe normaler Virulenz; als solche Nährmedien bezeichne ich:

1. sterile Kartoffelscheiben, 2. 10 Procent Weizenauszug,

3. je 5 Procent Quitten- und Eibischschleim,

4. festes Schafblutserum mit 25 Procent Traubenzuckerbouillon.

Darüber äussert sich Klett: „Allgemein gefasst würde sich also aus den Weil'schen Resultaten die Regel ableiten lassen, dass für das Zustandekommen der Sporenbildung nicht das aërobe Wachśthum, sondern die Art des Nährmaterials von ausschlaggebender Bedeutung ist."

Wenn ich an der Hand einwandsfreier Experimente nachgewiesen habe, dass bei strenger Anä̈robiose auf gewissen Nährmedien Sporenbildung eintritt, so folgt daraus, dass die Milzbrandbacillen absolut nicht des Sauerstoffes als solchen zur Sporenbildung bedürfen, sondern dass andere Oxydationsmittel, die die Bacillen anscheinend in gewissen Nährmedien vorfinden, bei strenger Anaërobiose dasselbe zu bewirken im Stande sind, was bei ärobem Wachsthum der Sauerstoff leistet.

Ich wies also bei anaërobem Wachsthum auf die Bedeutung des Nährmateriales für die Sporenbildung hin.

Wie kommt dann Klett zu der abermals unrichtigen Auffassung, es liesse sich aus meinen Resultaten die Regel ableiten, nicht das aërobe Wachsthum, sondern die Art des Nährmateriales sei für die Sporenbildung von ausschlaggebender Bedeutung?

Nun zu den Versuchen Klett's selbst.

Klett will unter streng anaëroben Bedingungen arbeiten und verwendet als Anaërobenapparate Buchner'sche Röhren, in denen sich die mit sporenfreiem Milzbrandmaterial geimpften Nährmedien befanden. Als solche verwendet er Schrägagar, erstarrtes Blutserum; von flüssigen: Bouillon, Quittensehleim und ähuliche. Nach seinen Angaben ist es absolut nicht wesentlich, die Nährmedien stets vor dem Impfen auszukochen. Er bebrütete die in Buchner'schen Röhren befindlichen geimpften Nährmedien und fand nach 2 Tagen, dass sich "trotz des anaëroben Wachsthums" massenhaft Sporen gebildet hatten.

Als Kriterium dafür, dass die Milzbrandbacillen unter streng anaëroben Bedingungen im inneren Reagensgläschen gewachsen sind, sieht Klett das Erlöschen eines brennenden Spahnes an, wenn derselbe nach vorsichtigem Oeffnen der Buchner'schen Röhre und nach Herausnahme des geimpften Reagircylinders in die äussere Buchner'sche Röhre hineingetaucht wurde.

Auf Grund des positiven Ausfalles der Sporenbildung in allen Nährmedien unter dem Einfluss von pyrogallolsaurem Kali kommt Klett zu dem Schlusse, dass zum Zustandekommen der Sporenbildung nicht die An- 
wesenheit von Sauerstoff erforderlich ist - das ist ja zufälliger Weise richtig -, dass dieselbe vielmehr auch unter sogenannten anaëroben Verhältnissen in einer Stickstoffatmosphäre regelmässig einzutreten pflegt.

Es waren allerdings nur sogenannte anaërobe Verhältnisse, die wir aber aërobe zu nennen gezwungen sind. Der Beweis für meine Behauptung soll gleich nachher erbracht werden.

Klett sagt wörtlich: Wie übrigens weiter unten (wo er die Luft durch den sehr leicht diffundirbaren Wasserstoff verdrängt) gezeigt wird, ist die geringe Menge von Sauerstoff, die z. B. in schon längere Zeit zubereiteten Agarröhrchen absorbirt ist, von sich aus keineswegs im Stande, unter anderen Bedingungen (in der Wasserstoffatmosphäre) eine Sporenbildung zu ermöglichen.

Wie kommt Klett zu einer Vorstellung über die Grösse des Luftvolumens, das durch den Wasserstoff verdrängt wurde?

Wer ofters Titrationen ausführt mit Phenolphtaleïn, das gegen Luft bezw. deren Kohlensäuregehalt empfindlich ist, kennt zur Genüge die erheblichen Differenzen, die sich ergeben, $j \theta$ nachdem man das betreffende Nährmedium durch Auskochen luftleer macht oder nicht. Wenn sich diese Angaben direct auch nur auf flüssige Nährmedien beziehen, so wird keinenfalls bestritten werden können, dass ein plastischer Körper, wie Agar, beim Erstarren mechanisch Luft einzuschliessen vermag, deren Menge zu unterschätzen wir keinen Grund haben. Man kann ja einwenden, diese Luftmenge könne nur minimal sein, da unsere exquisiten Anaërobier, wie Tetanus oder Rauschbrand, manchmal auch vortrefflich in nicht ausgekochten festen Nährmedien zu gedeihen vermögen.

Nachdem wir aber einerseits absolut keine Vorstellung darüber haben, welche directe Rolle dem Sauerstoff beim Zustandekommen bezw. Ausbleiben der Entwickelung in den Reinculturen unserer obligaten Anaërobier zukommt, nachdem andererseits Kedrow $\mathrm{ski}^{1}$ es für den Tetanusbacillus gezeigt hat, dass derselbe in einer Prodigiosuscultur trotz einer gesättigten Sauerstoffatmosphäre, hergestellt durch ununterbrochenes Einleiten von Sauerstoff, sich reichlich vermehrte, und dass hier der Sauerstoff nur das Zustandekommen eines Fermentes begünstigt, das dem Tetanusbacillus auch unter völlig aëroben Bedingungen das Gedeihen ermöglicht, nachdem ferner Kitt ${ }^{2}$ in grossen lufthaltigen Bouillonmengen auch prächtige Rauschbrandeulturen zu züchten vermochte, dürfen wir keinesfalls den positiven Ausfall des Wachsthumes eines strengen Anaërobiers als sicheres Kriterium dafür gelten lassen, dass das betreffende Nährmedium völlig sauerstofffrei war.

${ }^{1}$ Kedrowski, Diese Zeitschrift. Bd. XX. S. 358.

${ }^{2} \mathrm{Kitt}$, Centralblatt für Bakteriologie u. Parasitenkunde. Bd. XVII. S. 159. 
Dieser mechanisch im Nährmedium eingeschlossene Sauerstoff bildet aber nur einen Bruchtheil der Sauerstoffmenge, die den Milzbrandbacillen in den Buchner'schen Röhren zur Verfügung stand.

Das Klett'sche Reagens, das Erlöschen des brennenden Spahnes, soll aber, wie Klett behauptet, ein Kriterium nicht nur dafür sein, dass in der Buchner'schen Röhre kein Sauerstoff mehr vorhanden war, sondern sogar als Beweis dafür gelten, dass die Milzbrandbacillen in der inneren Reagensröhre unter streng anaëroben Bedingungen gewachsen wären.

Die Thatsachen der Physik und Chemie machen Front gegen den Werth eines solchen Indikators.

Da einerseits die Luft mit einem Drucke von $10333^{\mathrm{kg}}$ auf einem Quadratmeter Fläche lastet, andererseits es eine physikalische Fundamentalwahrheit ist, dass verschiedene Gase, die keine chemische Wirkung auf einander ausüben, durch Diffusion sich gleichförmig durch den ganzen Raum vertheilen, so muss in die geöffnete Buchner'sche Röhre, während das Proberöhrchen entnommen wurde, Sanerstoff eingedrungen sein und beweist das Erlöschen des brennenden Spahnes nur, dass sich in der Buchner'schen Röhre keine 21 Procent Sauerstoff und mehr als 79 Procent Stickstoff befanden.

\section{Eigene Versuche.}

\section{Colorimetrisch-chemischer Sauerstoffnachweis.}

In 10 Buchner'sche Röhren gab ich je 1 grm Pyrogallol und $10 \mathrm{~cm}$ ausgekochter 1 procent. KOH, ferner je ein Schrägagar- und je ein steriles Reagensröhrchen; letzteres war gefüllt mit reinem Pyrogallol. Die wohl versehlossenen Apparate verweilten zweimal 24 Stunden bei $37^{\circ} \mathrm{C}$.

Fünf Röhren wurden dann nach einander geöffnet, in Nachahmung der Klett'schen Versuche das Agarröhrchen entfernt und in das Pyrogallolröhrchen mit der Pipette $1^{\mathrm{ccm}}$ kochend heisser 10 proc. Kalilauge gegeben. Alsdann wurden die Buchner'schen Röhren, die im ganzen kaum 10 Sekunden geöffnet waren, wieder wohl verschlossen. Die Pyrogallollösung in den 5 Röhrchen war nach 4 Minuten noch gleichmässig hellgelb, nach 5 bis 10 Minuten entstand eine dünne schwarzbraune Zone am oberen Rande, die allmählich durch weitere Diffusion des Sauerstoffes nach unten fortschritt.

Die übrigen 5 Röhren wurden nun ebenfalls rasch geöffnet und ohne Entfernung des Agarröhrehens dem reinweissen Pyrogallol die ausgekochte Kalilauge zugesetzt, worauf mit dem Gummistopfen nach kaum 4 Secunden die Buchner'schen Röhren wieder sorgfältig verschlossen wurden. Nach 30 Minuten ist das pyrogallolsaure Kali noch rein hellgelb, ein sicherer Beweis dafür, dass mit der heissen Kalilauge keine Luft mit übertragen wurde. Nach 40 Minuten ist, in allen Röhrchen eine schwarzbraune Zone zu beobachten, die von oben nach unten mit der fortschreitenden Diffusion des Sauerstoffes sich vergrössert. Nachdem so in allen Buchner'schen Röhren die Anwesenheit von Sauerstoff mit absoluter Sicherheit erwiesen, 
brachte ich in das Innere der 10 Röhren einen brennenden Spahn. Er erlischt sofort und beginnt an der Luft wieder zu glimmen.

Physikalisch und colorimetrisch-chemisch wäre die Werthlosigkeit des Klett'schen Reagens bewiesen. Es sei nun aber auch meine Aufgabe, quantitativ festzustellen, welche Sauerstoffmengen den Milzbrandbacillen in den Buchner'schen Röhren zur Verfügung standen.

\section{Gasanalytische Absorption und volumetrische Bestimmung} des Sauerstoffes.

8 Buchner'sche Röhren, in denen sich je ein Gramm Pyrogallol, $10^{\mathrm{cem}}$ ausgekochter $\mathrm{KOH}$ und ein Schrägagarröhrchen befanden, waren mit Gummistopfen verschlossen, in die je 2 Glasröhren passten, die selbst mit Gummischläuchen und Quetschbähnen luftdicht abgeschlossen waren. Jede einzelne Röhre war auf's Sorgfältigste mit geschmolzenem Paraffin gedichtet. Dem Thermostaten von $37^{\circ}$ wurden sie nach den unten angegebenen Zeiten entnommen und in je 2 der procentuale Sauerstoffgehalt ermittelt. Die Absorption ${ }^{I}$ wurde bei $21^{\circ} \mathrm{C}$. nach dem Vorgange Doyère's in besonderen Apparaten vorgenommen, durch Combination der Quecksilbergasbüretten mit den einfachen Gasabsorptionspipetten, in denen sich pyrogallolsaures Kali befand.

Der Sauerstoffgehalt betrug im Mittel:

\begin{tabular}{|c|c|c|c|c|c|c|c|c|}
\hline Tach & 2 sti & ndigem & Verweilen & bel & 37 & & 17 & \\
\hline$n$ & 6 & ", & $"$ & $"$ & $"$ & & $13 \cdot 4$ & $"$ \\
\hline$"$ & $20^{1 / 2}$ & $"$ & $"$ & $"$ & $"$ & & $4 \cdot 6$ & $"$ \\
\hline & 26 & $"$ & $"$ & & $"$ & , & 0 & \\
\hline
\end{tabular}

In den Buchner'schen Röhren steht den Milabrandbacillen mehr als 20 Stunden lang Sauerstoff zur Verfügung. Wie ich ${ }^{2}$ gezeigt habe, tritt die Sporenbildung bei Gegenwart von Sauerstoff im Temperaturoptimum schon nach 16 Stunden ein. Ist da die Anwesenheit von Sporen zu verwundern, wenn den Bacillen länger als 20 Stunden Sauerstoff geboten war?

Nun möchte ich nur noch citiren, was Buchner ${ }^{3}$ selbst über seine eigene Methode sagt: „Beträgt der Luftraum der äusseren Röhre $100 \mathrm{~cm}$, die Menge der Pyrogallussäure $1 \mathrm{grm}$, jene der Zehntelkalilange $10^{\mathrm{ccm}}$, so ist im Brutkasten bei $37^{\circ}$ die Sauerstoffabsorption nach 24 Stunden vollendet. Für rein theoretische Versuche, bei denen von vorn herein jede Spur von Sauerstoff auszuschliessen ist, muss ohnehin eine der bisher bekannten Methoden verwendet werden."

Und last not least behauptet Klett, dass die Sporenbildung regelmässig in einer Stickstoffatmosphäre einzutreten pflegt.

Ist denn Luft, der der Sauerstoff theilweise entzogen ist, eine Stickstoffatmosphäre? Das kann ein Gemenge sein von Stickstoff, Sauerstoff, Kohlensäure, Argon, Ammoniumcarbonat, Ammoniumnitrit, Chlornatrium

1 Hempel. Gasanalytische Methoden. 1900. S, 44, $133 \mathrm{ff}$.

2 Weil, a. a. 0 .

${ }^{3}$ Buchner, Centralblatt für Bakteriologie u. Parasitenteunde: Bd. IV. S. 150. 
und anderen Verbindungen. Hätte Klett reines Ammoniumnitrit erhitzt, den Stickstoff in den Bottkin'schen Apparat geleitet, bis alle Luft verdrängt war, dann wäre ihm eine einwandsfreie Stickstoffatmosphäre zur Verfügung gestanden, die ihn höchstwahrscheinlich veranlasst hätte, diesen ersten Theil seiner Publication nochmals einer genauen Nachprüfung zu unterziehen.

Der zweite Theil der Klett'schen Arbeit ist theoretisch einwandsfrei; Klett vertrieb die Luft in den geimpften Nährmedien durch chemisch reinen Wasserstoff; als Anaërobenapparate benutzt er nach der Roux-Heim'schen Methode präparirte Reagensgläser und. den Bottkin'schen Apparat.

Za Klett's Erstaunen unterblieb in der einwandsfreien Wasserstoffatmosphäre die Sporenbildung auf allen Nährmedien, auf denen vorher "bei scheinbar streng anaëroben Bedingungen" Sporen gebildet wurden, gänzlich. Nur auf Quitten- und Eibischschleim konnte er Sporen nach der rein objectiven biologischen Methode nachweisen.

Ich lasse die beiden hierher gehörigen Tabellen Klett's nebenstehend folgen.

Dareh diese Resultate, den stets positiven Ausfall der Sporenbildung in der Buchner'schen Röhre im Vergleich zu dem stets negativen in der einwandsfreien Wasserstoffatmosphäre, glanbt sich Klett zu dem Schlusse berechtigt, dass die Stickstoffatmosphäre (in Wirklichkeit der Sauerstoff) das Zustandekommen der Sporen begünstige, während der Wasserstoff, in dessen Atmosphäre auf den gebräuchlichen Nährmedien niemals Sporen gebildet werden, einen schädigenden Einfluss auf die Entwickelung der Milzbrandbacillen ausübt, weshalb der Wasserstoff den Bakterien (nicht nur den Milzbrandbacillen) gegenüber nicht das indifferente Gas ist, für welches er bislang noch gehalten wird.

Solche offenbar unrichtige Folgerungen können indess die Resultate eines Hauser, eines Frankland oder eines Fränkel, die durch mūhevolle experimentelle Arbeiten die Unschädlichkeit des Wasserstoffes den gebräuchlichen, auch noch so leicht zu Grunde gehenden Bakterien gegenüber nachgewiesen haben, nicht im mindesten beeinträchtigen.

Auf die Vorstellung Klett's; dass in flüssigen Nährmedien die Luft durch Wasserstoff nicht so gut verdrängt werden könne, wie in festen, da man die letzteren umzukehren vermag, will ich nicht näher eingehen.

Wenn ich beim Beginne meiner Erwiderung sagte, dass der Text in directem Widerspruch steht mit manchen in den Tabellen niedergelegten Daten, so erinnere ich nur an Tabelle IV.

Klett sagt, in der Wasserstoffatmosphäre werden von den Milzbrandbacillen keine Sporen gebildet. Wie verhält es sich mit der positiven Sporenbildung im Quittenschleim und im Eibischschleim? 
Tabelle $\mathrm{I}$.

Ausgangsmaterial: Herzblut einer an Milzbrand gestorbenen Maus. Zü̈chtung in Buchner'schen Röhren.

\begin{tabular}{|c|c|c|c|c|}
\hline & $\begin{array}{c}\mathrm{Cu} \\
\text { angelegt }\end{array}$ & \begin{tabular}{|c|}
$\mathrm{t} \mathrm{u} \mathrm{r}$ \\
untersucht
\end{tabular} & Entwickelung & Sporenbildung \\
\hline Agar & 13. X. & 16. X. & reichlich & reichlich \\
\hline Agar + 3 Tr. Na. snlfurosum & 13. X. & 16. X. & " & " \\
\hline Agar +2 Tr. Na. selenosum & 13. X. & 16. X. & , & ", \\
\hline Traubenzuckeragar & 3. V. & 5. V. & " & " \\
\hline Bouillon . . . . & 1. XI. & 3. XI. & mässig reichlich & zieml. reichlich \\
\hline Traubenzuckerbouillon & 3. V. & 5. $\nabla$. & " & " \\
\hline Bouillon + 3 Tr. Na. sulfur. & 1. XI. & 3. XI. & zieml. reichlich & , \\
\hline Quittenschleim : . . & 20.XII. & 22. XII. & " & " \\
\hline Eibischschleim . . . & 20. XII. & 22. XII. & , & " \\
\hline Blutserum erstarrt . . & 16. VI. & 19. VI. & mässig reichlich & mässig reichlich \\
\hline
\end{tabular}

Tabelle IV. Züchtung in Wasserstoff.

Ausgangspunkt: Sporenfreies Material aus dem Thierkörper.

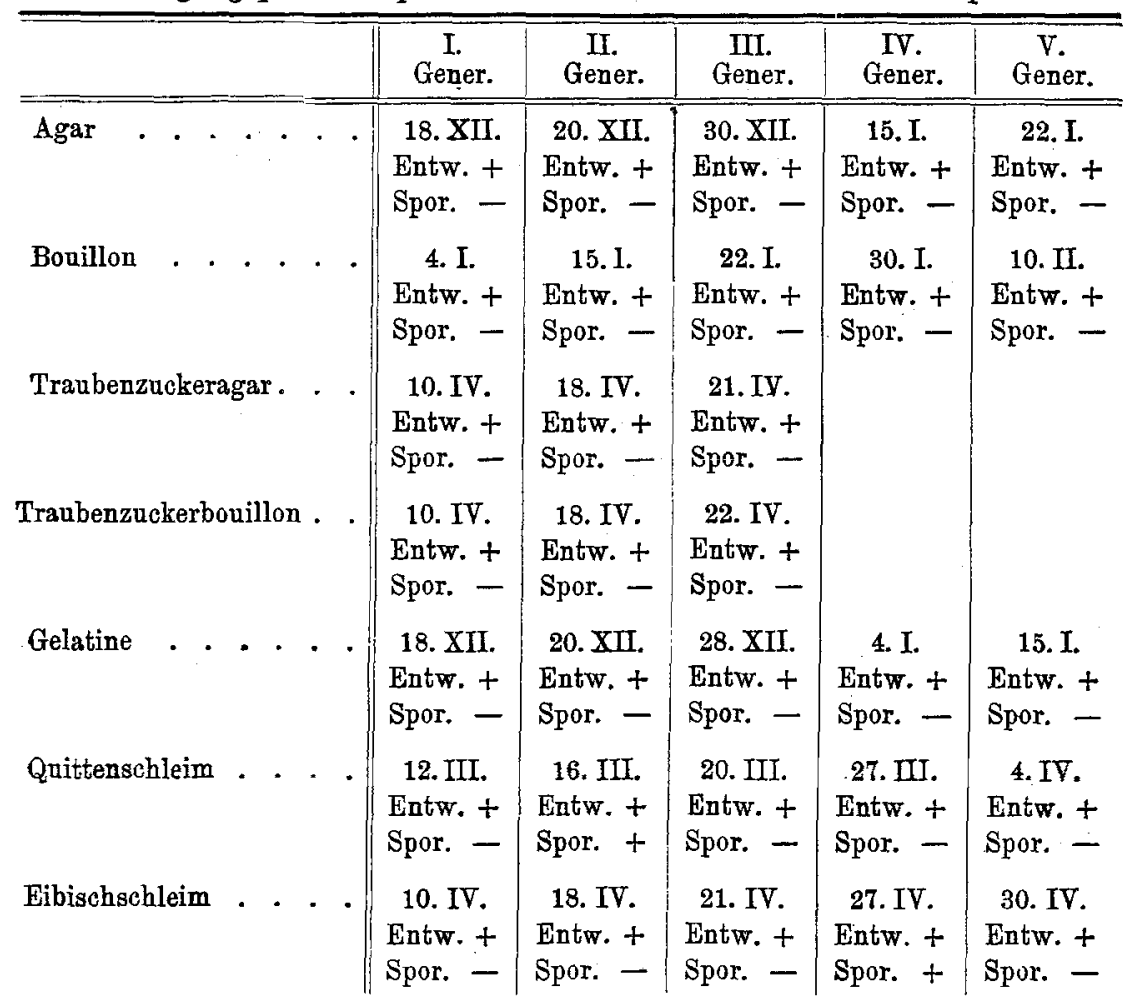


Will Klett behaupten, seine Versuche mit diesen beiden Nährmedien seien nicht einwandsfrei? Das zu beweisen, dürfte ihm schon deshalb schwer fallen, da die Resultate auf den übrigen in gleicher Weise behandelten Nährmedien, die ein einheitliches negatives Ergebniss zeigen, dagegen sprechen.

Wenn Klett einwendet, es ist doch nicht in allen Quitten- und Eibischschleimröhrchen Sporenbildung erfolgt, so frage ich: Ist denn den Individuen ein und derselben Bakterienart von der Natur ein mathematisch genaues Verhalten vorgeschrieben? Kennt nicht jeder Biologe das völig verschiedene Verhalten selbst der Nachlommen einer einzigen Bakterienzelle?

Einer unserer bedeutendsten Bakterienkenner, Migula ${ }^{1}$, betont in seiner Arbeit: „Ueber Abnahme und Regeneration der (aëroben) Sporenbildung bei Bakterien" die bedauernswerthe Thatsache, dass sehr viele Arten, die im Anfange reichlich Sporen bildeten, nach und nach bei fortgesetzter Züchtung immer mangelhafter Sporen bilden, so dass man schliesslich selbst in sehr alten Culturen nur noch ganz vereinzeltSporen findet.

Andererseits bin ich weit davon entfernt, zu behaupten, dass der wässerige Auszug von Quitten- und Eibischwurzeln etc. die natürliche Anlage der vegetativen Formen, Sporen zu bilden, derartig specifisch so zu beeinflussen vermag, dass jede vegetative Zelle des Milzbranderregers Dauerformen bilden muss. Es unterliegt keinem Zwreifel, dass der feine und complicirte Mechanismus der physiologischen Bedingungen der Sporenbildung direct von mehreren Factoren abhängig ist; als solche kennen wir bis jetzt: den günstigen Einfluss der Temperatur und des Nährmediums; an der dritten, von den Autoren gewöhnlich angeführten physiologisehen Bedingung der Sporenbildung ,einer ungehinderten Zufuhr von Sauerstoff " können wir, was die Milzbrandbacillen betrifft, nicht mehr festhalten.

Beim Studium der Sporenbildung unter streng anaëroben Bedingungen liess ich einwandsfreie Experimente entscheiden und begnügte mich mit der Constatirung der Thatsache, dass dieselbe auf unseren gebräuchlichen Nährböden ausbleibt, während es Nährböden giebt, auf denen die Milzbrandbacillen unter gleichen Bedingungen Sporen zu bilden vermögen, eine Thatsache, die ich zuerst experimentell festgestellt habe.

Wenn Klett in gesperrten Lettern sagt: Zum Zustandekommen der Sporenbildung beim Milzbrand ist nicht die Anwesenheit von Sauerstoff erforderlich, so bestätigt er die von mir festgestellte Thatsache. Von seinen eigenen Experimenten kann er zur Stütze der Bestätigung nur seine Quitten- und Eibischschleimversuche heranziehen.

${ }^{1}$ Migula, Zeitschrift für angew. Mikroskopie. Bd. V. 